\title{
Driving-induced population trapping and linewidth narrowing via the quantum Zeno
} effect

Christensen, Charles N.; Iles-Smith, Jake; Petersen, Torkil S.; Mørk, Jesper; McCutcheon, Dara P. S.

Published in:

Physical Review A (Atomic, Molecular and Optical Physics)

Link to article, DOI:

10.1103/PhysRevA.97.063807

Publication date:

2018

Document Version

Publisher's PDF, also known as Version of record

Link back to DTU Orbit

Citation (APA):

Christensen, C. N., lles-Smith, J., Petersen, T. S., Mørk, J., \& McCutcheon, D. P. S. (2018). Driving-induced population trapping and linewidth narrowing via the quantum Zeno effect. Physical Review A (Atomic, Molecular and Optical Physics), 97(6), [063807]. https://doi.org/10.1103/PhysRevA.97.063807

\section{General rights}

Copyright and moral rights for the publications made accessible in the public portal are retained by the authors and/or other copyright owners and it is a condition of accessing publications that users recognise and abide by the legal requirements associated with these rights.

- Users may download and print one copy of any publication from the public portal for the purpose of private study or research.

- You may not further distribute the material or use it for any profit-making activity or commercial gain

- You may freely distribute the URL identifying the publication in the public portal 


\title{
Driving-induced population trapping and linewidth narrowing via the quantum Zeno effect
}

\author{
Charles N. Christensen,,${ }^{1,}$ Jake Iles-Smith, ${ }^{1}$ Torkil S. Petersen, ${ }^{1}$ Jesper Mørk, ${ }^{1}$ and Dara P. S. McCutcheon ${ }^{2}$ \\ ${ }^{1}$ Department of Photonics Engineering, DTU Fotonik, Ørsteds Plads, 2800 Kongens Lyngby, Denmark \\ ${ }^{2}$ Quantum Engineering Technology Labs, H. H. Wills Physics Laboratory and Department of Electrical and Electronic Engineering, \\ University of Bristol, BS8 1FD, United Kingdom
}

(Received 5 July 2017; published 6 June 2018)

\begin{abstract}
We investigate the suppression of spontaneous emission from a driven three-level system embedded in an optical cavity via a manifestation of the quantum Zeno effect. Strong resonant coupling of the lower two levels to an external optical field results in a decrease of the decay rate of the third upper level. We show that this effect has observable consequences in the form of emission spectra with subnatural linewidths, which should be measurable using, for example, quantum dot-cavity systems in currently obtainable parameter regimes, and may find use in applications requiring the control of single-photon arrival times and wave-packet extent. These results suggest an underappreciated link between the Zeno effect, dressed states, and Purcell enhancement.
\end{abstract}

DOI: 10.1103/PhysRevA.97.063807

The quantum Zeno effect (QZE) refers to a collection of phenomena in which the evolution of a quantum system is inhibited by strong perturbations [1-5]. The first manifestation was coined and popularized by Sudarshan and Misra [5], where the effect is derived as a consequence of frequent projective measurements, i.e., wave-function collapses, which are shown to prevent the decay of an otherwise unstable state. Aside from being of general interest to those studying the theory of quantum measurement [6-11], the QZE may also constitute a valuable tool which could be used to inhibit decay and decoherence for quantum information applications [12].

Since the original formulation mentioned above, the QZE has since been attributed to other phenomena which deviate from the original in one or both of the following ways. They either (1) use strong unitary perturbations in the form of a constant coupling or a sequence of unitary "kicks" [13-16] and/or (2) they inhibit coherent dynamics, as opposed to inhibiting an incoherent irreversible process $[13,17]$. Experimentally, the QZE has been demonstrated in a manner closest to the original proposal in cold atom traps $[18,19]$ and superconducting qubits [20]. The first measurement attributed to the QZE, however, was made by Itano et al. [21], who used frequent measurements of a trapped ion to inhibit coherent evolution driven by an rf field, which actually places it in the second of the two categories above, along with those since performed on solid-state spins in diamond [22] and cold atom clouds [23]. Experiments falling into the first category above most notably include dynamical decoupling schemes [24,25], which make use of unitary pulse sequences to prolong coherence times.

Although all these phenomena have been referred to as the QZE, it should be understood that the physical concepts invoked vary considerably, leaving the true essence of the QZE somewhat obscure. In particular, as has long been noted [6-10], the inhibition of coherent dynamics requires no notion of wave function collapse, and can in fact be derived from

\footnotetext{
*Corresponding author: charles.n.chr@gmail.com
}

uncontroversial, purely dynamical arguments. On the other hand, a decay process modeled as exponential and strictly Markovian cannot be inhibited by strong unitary coupling, nor by projective measurements [26,27]. Thus, any process that resembles the QZE (the inhibition of some decay process) must exploit the short-time coherent and reversible nature of that process $[1,6]$. In this case, however, concepts of measurement and wave function collapse need not be invoked, meaning these cannot be the essential ingredients in the general case. The only consistent definition of the QZE that encompasses all the effects described above is that it is one in which shorttime nonexponential behavior is exploited by some external perturbation to change overall decay rates.

In this work we adopt this generalized definition of the QZE and elucidate a manifestation which exploits the frequency domain counterpart of coherent short-time behavior, namely the finite bandwidth of the environment into which decay takes place [15,16,26-28]. This approach requires only easily implemented constant coherent couplings and highlights an underappreciated link between the QZE and the well-known concepts of dressed states and Purcell enhancement. Our findings are phrased in terms of a three-level system embedded in a moderate $Q$-factor optical cavity, which could be experimentally realized by, e.g., a resonantly driven semiconductor quantum dot in a photonic crystal cavity [29-32], as envisaged in Fig. 1(a). We show that strong driving of the lower two levels results in population trapping in the upper level. This has a clear experimental signature in the form of emission spectra with linewidths which narrow with increasing coupling strength, and we show that this manifestation of the QZE should be experimentally accessible with current technologies.

It is instructive to first consider different phenomena which may take place in a three-level system and how they relate to the QZE. In Fig. 1(b) we consider the simple case studied in Ref. [33], which consists of three equally spaced levels $|g\rangle,|e\rangle$, and $|p\rangle$. If states $|p\rangle$ and $|e\rangle$ are coherently coupled, for example with a resonant laser with Rabi frequency $\Omega_{p e}$, a system initially in $|\psi(0)\rangle=|p\rangle$ will evolve into 


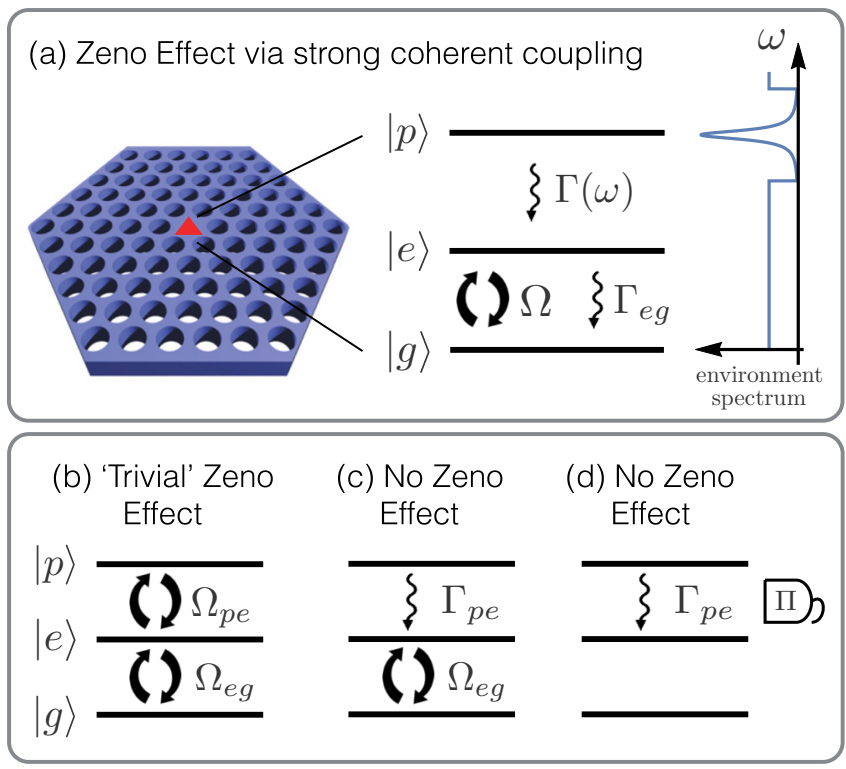

FIG. 1. (a) Three-level system embedded in an optical cavity such that the environment spectrum has a maximum resonant with the $|p\rangle \rightarrow|e\rangle$ transition. Coherent coupling between the lower two $|e\rangle$ and $|g\rangle$ levels inhibits population decay from the upper $|p\rangle$ level via a manifestation of the Quantum Zeno effect. (b) A similar system with two coherent couplings as shown. When $\Omega_{e g} \gg \Omega_{p e}$ the system exhibits a "trivial" Zeno effect with population residing predominately in $|p\rangle$. Parts (c) and (d) consider a phenomenological incoherent decay process from $|p\rangle$ to $|e\rangle$, with the addition of coherent coupling (c) or projective state population measurements (d), neither of which give rise to any Zeno effect.

$|\psi(t)\rangle=\cos \left(\Omega_{p e} t / 2\right)|p\rangle+\sin \left(\Omega_{p e} t / 2\right)|e\rangle \quad$ [34], with the probability to remain in the initial state given by $P(t)=$ $|\langle p \mid \psi(t)\rangle|^{2}=\cos ^{2}\left(\Omega_{p e} t / 2\right)$. If a second field of strength $\Omega_{e g}$ is introduced which couples $|g\rangle$ and $|e\rangle$, the probability to remain in the initial state becomes $|\langle p \mid \psi(t)\rangle|^{2}=\left[\left(\Omega_{e g}^{2}+\right.\right.$ $\left.\left.\Omega_{p e}^{2} \cos \left(\Omega_{R} t / 2\right)\right) / \Omega_{R}^{2}\right]^{2}$ with $\Omega_{R}^{2}=\Omega_{p e}^{2}+\Omega_{e g}^{2}$. Evidently, if $\Omega_{e g} \gg \Omega_{p e}$ the transfer of population to $|e\rangle$ is inhibited by the strong coupling of $|e\rangle$ to $|g\rangle$. Although we may refer to such a process as the QZE, since a strong perturbation inhibits a population transfer, it is a consequence of nothing more than the Hamiltonian dynamics of the coupled three levels, having various classical analogs [35]. This simple model highlights the triviality of the QZE when referring to the inhibition of a process coherent on all time scales.

We now consider Fig. 1(c), in which we replace the coherent interaction between $|p\rangle$ and $|e\rangle$ with a fixed exponential decay rate $\Gamma_{p e}$. One now finds that regardless of any coupling between the lower states, the probability for the excitation to remain in $|p\rangle$ is $P(t)=e^{-\Gamma_{p e} t}$; in contrast to case (b), a strong coherent drive no longer affects the rate of population transfer from state $|p\rangle$. Finally, we consider Fig. 1(d), where strong measurements project onto one of the three states [27,36]. The probability that the excitation remains in $|p\rangle$ after a time $t$ is then calculated by splitting $t$ into $N$ intervals of $\Delta t=t / N$, after each of which we perform a measurement. Now we find $P(t)=P(\Delta t)^{N}=e^{-\Gamma_{p e} t}$, the same result as before. This demonstrates that even rapid projective measurements cannot inhibit a purely exponential decay process.

What then is necessary for a system to display a nontrivial QZE? We will see that the essential condition is that the environment into which decay takes place must have a structured frequency spectrum. To see this, let us now consider the model in Fig. 1(a). It consists of a three-level system, with for now only the upper two states coupled to an electromagnetic environment, modeled as a reservoir of harmonic oscillators. The emission suppression we describe below does not necessitate a system with three levels, though with three we can consider the lower two levels as being driven by a continuous-wave laser of frequency $\omega_{l}$ and Rabi frequency $\Omega$, which does not directly couple to $|p\rangle$. We set $\Omega=0$ when necessary to consider the undriven case. The total Hamiltonian is (we set $\hbar=1$ ) [37] $H=H_{S}+H_{I}+H_{E}$ with

$$
H_{S}=\omega_{p}|p\rangle\left\langle p\left|+\omega_{e}\right| e\right\rangle\langle e|+\Omega \cos \left(\omega_{l} t\right)\left(\sigma_{e g}^{\dagger}+\sigma_{e g}\right),
$$

while $H_{E}=\sum_{\mathbf{k}} \omega_{\mathbf{k}} b_{\mathbf{k}}^{\dagger} b_{\mathbf{k}}, H_{I}=\sum_{\mathbf{k}} g_{\mathbf{k}} \sigma_{p e}^{\dagger} b_{\mathbf{k}}+$ H.c., where $\omega_{e}$ and $\omega_{p}$ are the energies of $|e\rangle$ and $|p\rangle, \sigma_{e g}=$ $|g\rangle\langle e|$ and $\sigma_{p e}=|e\rangle\langle p|$, and $b_{\mathbf{k}}^{\dagger}$ is the creation operator for a photon with wave vector $\mathbf{k}$, frequency $\omega_{\mathbf{k}}$, and coupling constant $g_{\mathbf{k}}$. Moving into a rotating frame with $T(t)=\exp \left[i \omega_{l}(|e\rangle\langle e|+2| p\rangle\langle p|) t\right]$ and making a rotating wave approximation, valid when $\Omega \ll$ $\omega_{e}$ and $\omega_{l} \approx \omega_{e}$ [38], we find $H^{\prime}(t)=H_{S}^{\prime}+H_{I}^{\prime}(t)+$ $H_{E}$, where $H_{S}^{\prime}=\Delta|p\rangle\langle p|+(\Omega / 2)\left(\sigma_{e g}^{\dagger}+\sigma_{e g}\right)$ and $H_{I}^{\prime}(t)=$ $\sum_{\mathbf{k}} g_{\mathbf{k}}\left(\sigma_{p e}^{\dagger} b_{\mathbf{k}} e^{i \omega_{e} t}+\right.$ H.c. $)$, where we have set the laser resonant with the $|e\rangle \rightarrow|g\rangle$ transition, $\omega_{l}=\omega_{e}$, and defined $\Delta=\omega_{p}-2 \omega_{e}$ as the level spacing asymmetry. In the interaction picture we have $\tilde{H}_{I}^{\prime}(t)=U_{0}^{\dagger}(t) H_{I}^{\prime}(t) U_{0}(t)$ with $U_{0}(t)=$ $\exp \left[-i\left(H_{S}^{\prime}+H_{E}\right) t\right]$.

Now, beginning from an ansatz of the form

$$
|\psi\rangle=\alpha(t)|p\rangle|\phi\rangle+\sum_{\mathbf{k}}\left(\beta_{\mathbf{k}}(t)|+\rangle+\gamma_{\mathbf{k}}(t)|-\rangle\right) b_{\mathbf{k}}^{\dagger}|\phi\rangle,
$$

where $| \pm\rangle=(1 / \sqrt{2})(|e\rangle \pm|g\rangle)$ and $|\phi\rangle$ denotes the vacuum, it can be shown that $\dot{\alpha}(t)=-\int_{0}^{t} d t^{\prime} \alpha\left(t^{\prime}\right) \mathcal{K}\left(t-t^{\prime}\right)$, where the dissipation kernel is given by [39]

$$
\mathcal{K}(t)=\int_{-\infty}^{\infty} d \omega \Gamma(\omega) e^{-i \omega t} e^{i\left(\omega_{p}-\omega_{e}\right) t} \cos (\Omega t / 2) / \pi,
$$

and $\Gamma(\omega)=\pi \sum_{\mathbf{k}} g_{\mathbf{k}}^{2} \delta\left(\omega_{\mathbf{k}}-\omega\right)$ is the spectral density. Moving to Laplace variables defined by $\bar{\alpha}(s)=\int_{0}^{\infty} d t e^{-s t} \alpha(t)$, we find $\bar{\alpha}(s)=\alpha(0)[s+\overline{\mathcal{K}}(s)]^{-1}$, which contains the full nonMarkovian decay dynamics of the $|p\rangle$ state. Taking a spectral density corresponding to a Lorentzian cavity $[27,40,41]$ $\Gamma(\omega)=\Gamma_{\text {cav }}(\kappa / 2)^{2}\left[\left(\omega-\omega_{c}\right)^{2}+(\kappa / 2)^{2}\right]^{-1}$, where $\kappa$ and $\omega_{c}$ are the cavity width and central frequency, respectively, the Laplace space response kernel can be found to be $\overline{\mathcal{K}}(s)=$ $\Gamma_{\text {cav }}(\kappa / 2)(s+\kappa / 2)\left[(s+\kappa / 2)^{2}+(\Omega / 2)^{2}\right]^{-1}$, where we have set the cavity resonant with the $|p\rangle \rightarrow|e\rangle$ transition, $\omega_{c}=$ $\omega_{p}-\omega_{e}$. The Markovian limit can be found by setting $s=0$ in $\overline{\mathcal{K}}(s)$, and for zero driving we find exponential decay $|\alpha(t)|^{2}=$ $P_{\mathrm{M}}(t)=\exp \left[-2 \Gamma_{\text {cav }} t\right]$.

The original QZE arises by assuming that we monitor the occupation of $|p\rangle$ on a time scale $\Delta t$ short enough that $P(\Delta t)$ can be expanded to lowest order, before the Markovian limit 
is reached. From the expressions above we find $P(\Delta t) \approx 1-$ $\Delta t^{2} \Gamma_{\text {cav }} \kappa / 2$, and for a total time $t=N \Delta t$ and large $N$ we then find the probability

$$
P(t)=P(\Delta t)^{N} \approx \exp \left[-2 \frac{\kappa}{4 \omega_{m}} \Gamma_{\mathrm{cav}} t\right],
$$

where $\omega_{m}=1 / \Delta t$ is the measurement frequency. We see that if $\omega_{m}$ is sufficiently larger than the environment bandwidth $\kappa$, then the decay is suppressed compared to the Markovian value $P_{\mathrm{M}}(t)$. Conversely, as $\kappa \rightarrow \infty, P(\Delta t)=P_{\mathrm{M}}(\Delta t)$ cannot be expanded in the same way, and $P(t)=P_{M}(\Delta t)^{N}=P_{\mathrm{M}}(t)$ regardless of $\omega_{m}$.

However, there is another way the finite bandwidth of the environment can be exploited to inhibit decay, namely by strongly driving the system. For nonzero $\Omega$, in the Markovian limit we find

$$
P_{\mathrm{M}}(t)=\exp \left[-2 \frac{\kappa^{2}}{\kappa^{2}+\Omega^{2}} \Gamma_{\text {cav }} t\right],
$$

where now the Rabi frequency $\Omega$ replaces the role of the measurement frequency, and it is this which must be larger than the environment bandwidth to suppress the decay rate. In both of these cases, we may attribute the decay inhibition to a strong external perturbation (either measurements or continuous driving), which interrupts the otherwise efficient transfer of population to the environment. This inhibition fails in the limit of infinite environment bandwidth $\kappa \rightarrow \infty$, as the environment response time then tends to zero, and is necessarily faster than any external perturbation can act.

To elucidate this manifestation of the QZE further, we now develop a detailed analysis based on a Born-Markov master equation, which also allows us to explore clear experimental signatures of this driving-induced QZE. The master equation describes the three-level system reduced density operator $\rho(t)$, treating $H_{I}^{\prime}(t)$ as a perturbation to second order, and to which we add a term $\sum_{\mathbf{k}} g_{\mathbf{k}} \sigma_{e g}^{\dagger} b_{\mathbf{k}} e^{i \omega_{e} t}+$ H.c. to include $|e\rangle \rightarrow|g\rangle$ emission. In the Schrödinger picture we have [42-44]

$$
\begin{aligned}
\dot{\rho}(t)= & -i\left[H_{S}^{\prime}, \rho(t)\right]-\int_{0}^{\infty} d \tau \operatorname{Tr}_{E} \\
& \times\left[H_{I}^{\prime}(t),\left[U_{0}(\tau) H_{I}^{\prime}(t-\tau) U_{0}^{\dagger}(\tau), \rho(t)|\phi\rangle\langle\phi|\right]\right] .
\end{aligned}
$$

Neglecting Lamb shift terms and assuming independent fluctuations causing $|p\rangle \rightarrow|e\rangle$ and $|e\rangle \rightarrow|g\rangle$ emission, we find the master equation $\dot{\rho}(t)=-i\left[H_{S}^{\prime}, \rho(t)\right]+\mathcal{D}_{p e}[\rho(t)]+$ $\mathcal{D}_{e g}[\rho(t)]$, where

$$
\mathcal{D}_{p e}[\rho]=\sum_{\{\eta\}_{p e}} \Gamma(\eta)\left(\left[\sigma_{p e}, \rho A_{p e}^{\dagger}(\eta)\right]-\left[\sigma_{p e}^{\dagger}, A_{p e}(\eta) \rho\right]\right),
$$

with $\quad A_{p e}(\eta) \quad$ satisfying $\quad U_{S}(s) \sigma_{p e} U_{S}^{\dagger}(s) e^{i \omega_{e} s}=$ $\sum_{\{\eta\}_{p e}} e^{i \eta s} A_{p e}(\eta)$ and $\sum_{\{\eta\}_{p e}} A_{p e}(\eta)=\sigma_{p e}$, and for this term $\{\eta\}_{p e}=\left\{\omega_{p}-\omega_{e} \pm \Omega / 2\right\}$. The second dissipator is of precisely the same form, but with all occurrences of $\sigma_{p e}$ replaced with $\sigma_{e g}$ and the summation running over $\{\eta\}_{e g}=\left\{\omega_{e}, \omega_{e} \pm \Omega\right\}$. In the context of our master equation, the QZE is now seen through the rates $\Gamma(\eta)$, which sample the spectral density at frequencies which depend on the driving strength $\Omega[26,28]$. From these frequency-dependent rates one can see that in general emission can be enhanced as well as suppressed, just as measurements can lead to both the Zeno effect and the anti-Zeno effect $[1,28,36]$. In the following we assume $\Gamma(\eta)$ is Lorentzian and resonant in the vicinity of the $|p\rangle \rightarrow|e\rangle$ transition as above (leading to spontaneous emission suppression), but takes on a constant value $\Gamma_{e g}$ in the vicinity of the $|e\rangle \rightarrow|g\rangle$ transition.

We now consider the emission spectrum of the system when initializing in $|p\rangle$ and driving the lower two levels. For concreteness we use parameters which correspond to quantum dots in photonic crystal cavities [29-31], for which the states $|e\rangle$ and $|p\rangle$ could be formed by the exciton and biexciton, with the level spacing asymmetry $\Delta$ then the biexciton binding energy [45]. Some care must be taken to choose an appropriate measurement time interval, since the emitted field will be neither stationary nor vanishing in the long-time limit. We therefore consider the time-dependent spectrum defined as [46-48]

$$
R(\Delta \omega, t)=\operatorname{Re}\left[\int_{0}^{t} d s \int_{0}^{t-s} d \tau g^{(1)}(s, \tau) e^{(\nu-i \Delta \omega) \tau} e^{-2 v(t-s)}\right],
$$

where $v$ is the resolution of the spectrometer, assumed to be Lorentzian, $\Delta \omega=\omega-\omega_{l}$ the difference between the laser frequency $\omega_{l}$ and the absolute emission frequency $\omega$, and $g^{(1)}(t, \tau)=\left\langle E(t+\tau) E^{\dagger}(t)\right\rangle$ is the first-order field correlation function with $E(t)$ the positive frequency component of
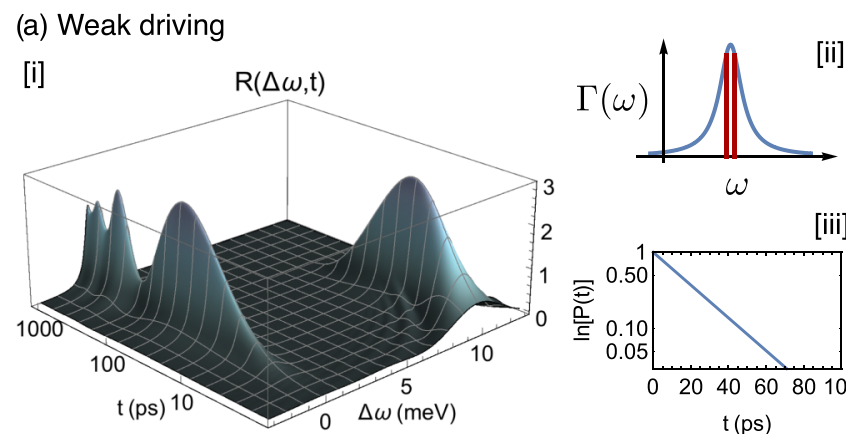

[ii]

(b) Strong driving
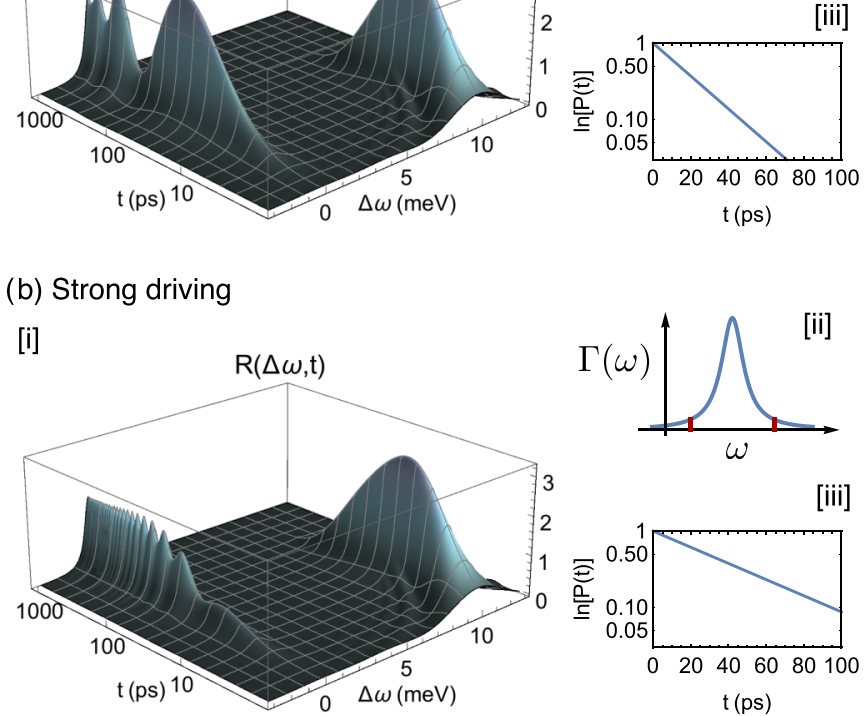

[iii]

FIG. 2. Parts [i] show time-dependent emission spectra for a three-level system initially in the upper state $|p\rangle$, with driving between the lower two levels being (a) weak $(\Omega=10 \mu \mathrm{eV})$ and (b) strong $(\Omega=100 \mu \mathrm{eV})$. For (b) emission from the lower subsystem is delayed, due to a decrease in the emission rates for the upper system, as indicated by the red lines in [ii], and shown explicitly by the population dynamics of the $|p\rangle$ level in [iii]. Parameters: $a=b=1, \Delta=10 \mathrm{meV}, \kappa=0.1 \mathrm{meV}, \Gamma_{e g}^{-1}=$ $500 \mathrm{ps},\left[2 \Gamma_{\text {cav }}\right]^{-1}=20 \mathrm{ps}$, and $v^{-1}=2 \mathrm{ps}$. 
(a) Weak driving
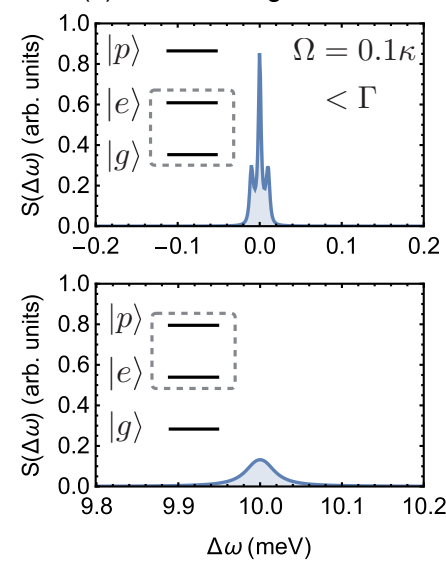

(b) Strong driving
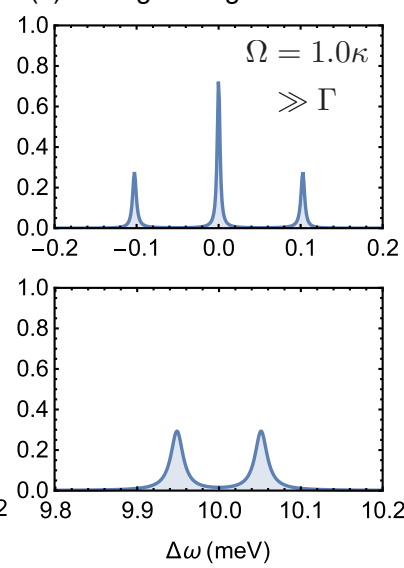

(c) Very strong driving
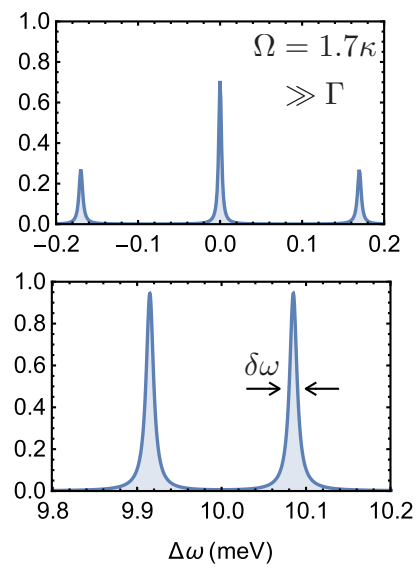

(d)

(e)
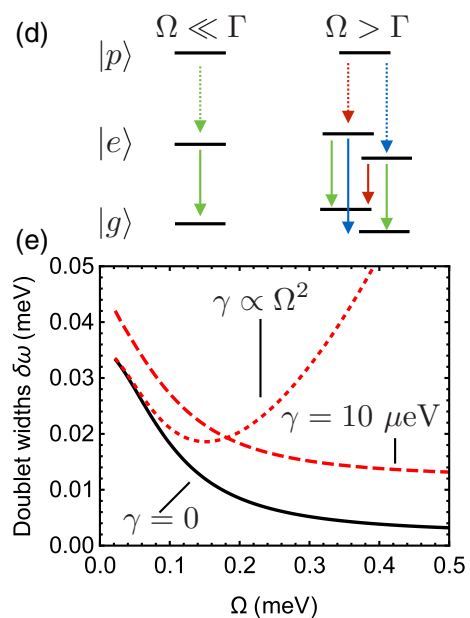

FIG. 3. Parts (a)-(c) show time-integrated emission spectra for weak $(\Omega=10 \mu \mathrm{eV})$, $\operatorname{strong}(\Omega=100 \mu \mathrm{eV})$ and very strong $(\Omega=170 \mu \mathrm{eV})$ driving strengths, with the two rows showing features around $\Delta \omega=0$ and $\Delta \omega=10 \mathrm{meV}$, pertaining to the two subsystems as indicated. The driving hybridizes the $|e\rangle$ and $|g\rangle$ levels into dressed states as depicted in (d), which then splits the $|p\rangle$ emission line into a doublet, whose peaks then narrow with increasing $\Omega$, as seen by the solid curve in (e). The red dashed curve in (e) shows the effect of a constant pure-dephasing term, while the red dotted curve corresponds to a driving-dependent dephasing term as expected for excitons in quantum dots. Here $v=0.3 \mu \mathrm{eV}$ and all other parameters as in Fig. 2.

the electric field. The correlation function is calculated by making the identification $E^{\dagger}(t) \propto a \sigma_{e g}(t)+b \sigma_{p e}(t)$ with $a$ and $b$ constants, and can then be calculated using our master equation and the quantum regression theorem $[37,43,46]$.

We first consider the case in which the spectrometer is rather broad compared to typical spectral features of the driven threelevel system, corresponding to the condition $v>\Gamma\left(\omega_{c}\right), \Omega$, the benefit being that the spectrometer can temporally resolve the system dynamics. In Fig. 2 we show time-dependent spectra for a spectrometer resolution of $v^{-1}=2 \mathrm{ps}$, for weak (a) and strong (b) driving, where the bare $|p\rangle \rightarrow|e\rangle$ transition is $\Delta=10 \mathrm{meV}$ larger than the $|e\rangle \rightarrow|g\rangle$ transition. The spectra show an initial $\sim 1$ ps delay due to "filling" of the spectrometer [46], giving way to emission from $|p\rangle$ seen by the peak around $\Delta \omega=10 \mathrm{meV}$. This is followed by emission from $|e\rangle$ seen around $\Delta \omega=0$, in which damped Rabi oscillations can be resolved. Comparing (a) and (b), we see that stronger driving introduces a greater delay before emission from $|e\rangle$ is observed. This is because the decay rate of the $|p\rangle$ level decreases, as seen explicitly in parts [iii] which show the population dynamics.

We now consider the case where $v \ll \Gamma\left(\omega_{c}\right), \Omega$. In this limit the spectrometer is able to resolve detailed spectral features, but provides little timing resolution, and it is therefore more appropriate to consider the time-integrated spectrum $S(\Delta \omega)=\int_{0}^{T} d t R(\Delta \omega, t)$ for $T \gg v^{-1}$. This is what would typically be measured experimentally when using a highresolution Fabry-Pérot interferometer [46]. In Figs. 3(a)3(c) we show time-integrated spectra for increasing driving strengths as indicated, for a spectrometer with realistic resolution $v=0.3 \mu \mathrm{eV}=2 \pi \times 67 \mathrm{MHz}[49,50]$, and an integration time of $T=3 \mathrm{~ns}$. With this increased spectral resolution, we can now see that the driving causes the emission peak from the $|p\rangle$ level to split into a doublet, while simultaneously the spectral features around $\Delta \omega=0$ pertaining to the lower two levels display a Mollow triplet. As depicted in Fig. 3(d), the driving hybridizes the lower two levels into dressed states, giving the upper $|p\rangle$ level two decay paths of differing energies. These paths sample the spectral density away from its peak centered at the bare undressed $|p\rangle \rightarrow|e\rangle$ transition energy, and have correspondingly suppressed rates. The suppression of the rates with increased driving strength, which can be considered the QZE, here manifests as a narrowing of the emission lines. This narrowing is clearly seen in Fig. 3(e), where the solid black curve shows the doublet peak linewidths $\delta \omega$ as a function of driving strength.

Also shown in Fig. 3(e) is the behavior of the doublet linewidths when dephasing is present, obtained by adding a term $2 \gamma(|e\rangle\langle e|\rho| e\rangle\langle e|-(1 / 2)\{|e\rangle\langle e|, \rho\})$ to the master equation defined in Eq. (6) and shown by the dashed red curve. For the specific case of excitons in quantum dots, excitationinduced dephasing caused by coupling to phonons is expected to give a driving-dependent dephasing rate $\gamma \approx \pi \alpha k_{B} T \Omega^{2}$ [49-51]. For a realistic exciton-phonon coupling constant of $\alpha=0.03 \mathrm{ps}^{2}$ and temperature of $T=4 \mathrm{~K}$ we obtain the dotted red curve. Importantly, due to the quadratic nature of driving dependence, a clear reduction in the doublet peak width is still observed, after which the dephasing overwhelms the suppression of spontaneous emission and the peaks begin to broaden. We note, however, that even in this regime spontaneous emission is still suppressed.

We have shown that strong driving of a three-level system in an optical cavity can give rise to spontaneous emission suppression via a manifestation of the quantum Zeno effect. This effect can also be interpreted as the result of the drivinginduced dressed states of the system sampling the structured spectral density away from its peak. Viewed in this way, the effect we observe is in close analogy with the well-known Purcell effect. We stress that this alternate interpretation, rather than invalidating the first, in fact points towards the ubiquity of quantum Zeno dynamics when it refers to the combination of external controls and finite bandwidth environmental interactions to control spontaneous emission. 
J.I.-S. and J.M. acknowledge support from the Danish Research Council (Grant No. DFF-4181-00416) and Villum Fonden (NATEC Centre). This project has received funding from the European Union's Horizon 2020 research and innovation programme under the Marie Skłodowska-Curie grant agreement No. 703193.
[1] P. Facchi and S. Pascazio, J. Phys. A: Math. Theor. 41, 493001 (2008).

[2] Y.-R. Zhang and H. Fan, Sci. Rep. 5, 11509 (2015).

[3] W. M. Itano, J. Phys.: Conf. Ser. 196, 012018 (2009).

[4] P. Facchi, G. Marmo, and S. Pascazio, J. Phys.: Conf. Ser. 196, 012017 (2009).

[5] B. Misra and E. C. G. Sudarshan, J. Math. Phys. 18, 756 (1977).

[6] T. Petrosky, S. Tasaki, and I. Prigogine, Phys. Lett. A 151, 109 (1990).

[7] E. Block and P. R. Berman, Phys. Rev. A 44, 1466 (1991).

[8] S. Pascazio and M. Namiki, Phys. Rev. A 50, 4582 (1994).

[9] L. E. Ballentine, Phys. Rev. A 43, 5165 (1991).

[10] Q. Ai, D. Xu, S. Yi, A. G. Kofman, C. P. Sun, and F. Nori, Sci. Rep. 3, 1752 (2013).

[11] T. M. Stace and S. D. Barrett, Phys. Rev. Lett. 92, 136802 (2004).

[12] G. A. Paz-Silva, A. T. Rezakhani, J. M. Dominy, and D. A. Lidar, Phys. Rev. Lett. 108, 080501 (2012).

[13] P. Facchi and S. Pascazio, Irreversible Quantum Dynamics (Springer, Berlin, Heidelberg, 2003), pp. 141-156.

[14] P. Facchi, D. A. Lidar, and S. Pascazio, Phys. Rev. A 69, 032314 (2004).

[15] A. G. Kofman and G. Kurizki, Phys. Rev. Lett. 87, 270405 (2001).

[16] A. G. Kofman and G. Kurizki, Phys. Rev. Lett. 93, 130406 (2004).

[17] P. Facchi and S. Pascazio, Phys. Rev. Lett. 89, 080401 (2002).

[18] M. C. Fischer, B. Gutiérrez-Medina, and M. G. Raizen, Phys. Rev. Lett. 87, 040402 (2001).

[19] Y. S. Patil, S. Chakram, and M. Vengalattore, Phys. Rev. Lett. 115, 140402 (2015)

[20] P. M. Harrington, J. T. Monroe, and K. W. Murch, Phys. Rev. Lett. 118, 240401 (2017).

[21] W. M. Itano, D. J. Heinzen, J. J. Bollinger, and D. J. Wineland, Phys. Rev. A 41, 2295 (1990).

[22] J. Wolters, M. Strauß, R. S. Schoenfeld, and O. Benson, Phys. Rev. A 88, 020101 (2013).

[23] F. Schäfer, I. Herrera, S. Cherukattil, C. Lovecchio, F. S. Cataliotti, F. Caruso, and A. Smerzi, Nat. Commun. 5, 3194 (2014).

[24] L. Viola and S. Lloyd, Phys. Rev. A 58, 2733 (1998).

[25] L.-A. Wu and D. A. Lidar, Phys. Rev. Lett. 88, 207902 (2002).

[26] A. G. Kofman and G. Kurizki, Acta Phys. Slov. 49, 541 (1999).

[27] G. Harel, A. G. Kofman, A. Kozhekin, and G. Kurizki, Opt. Express 2, 355 (1998).

[28] A. G. Kofman and G. Kurizki, Nature (London) 405, 546 (2000).

[29] D. Englund, D. Fattal, E. Waks, G. Solomon, B. Zhang, T. Nakaoka, Y. Arakawa, Y. Yamamoto, and J. Vučković, Phys. Rev. Lett. 95, 013904 (2005).
[30] K. Hennessy, A. Badolato, M. Winger, D. Gerace, M. Atature, S. Gulde, S. Falt, E. L. Hu, and A. Imamoglu, Nature (London) 445, 896 (2007).

[31] K. Roy-Choudhury and S. Hughes, Phys. Rev. B 92, 205406 (2015).

[32] K. Roy-Choudhury and S. Hughes, Opt. Lett. 40, 1838 (2015).

[33] A. Peres, Am. J. Phys. 48, 931 (1980).

[34] D. Walls and G. Milburn, Quantum Optics (Springer, New York, 2008).

[35] For example, two weakly coupled classical oscillators will exchange energy, which can be inhibited by strongly coupling one of the oscillators to a large mass [33].

[36] A. G. Kofman and G. Kurizki, Phys. Rev. A 54, R3750 (1996).

[37] A. Nazir and D. P. S. McCutcheon, J. Phys.: Condens. Matter 28, 103002 (2016).

[38] M. Grifoni and P. Hänggi, Phys. Rep. 304, 229 (1998).

[39] Here we have extended the lower integration limit from zero to $-\infty$, which is valid when $\omega_{c} / \kappa \ll 1$, and typically very well satisfy for optical cavities [40,41].

[40] T. Suhr, N. Gregersen, K. Yvind, and J. Mørk, Opt. Express 18, 11230 (2010).

[41] T. Suhr, N. Gregersen, M. Lorke, and J. Mørk, Appl. Phys. Lett. 98, 211109 (2011)

[42] H. P. Breuer and F. Petruccione, The Theory of Open Quantum Systems (Oxford University Press, Oxford, 2002).

[43] D. P. S. McCutcheon, Phys. Rev. A 93, 022119 (2016).

[44] K. Roy-Choudhury and S. Hughes, Optica 2, 434 (2015).

[45] F. Hargart, M. Müller, K. Roy-Choudhury, S. L. Portalupi, C. Schneider, S. Höfling, M. Kamp, S. Hughes, and P. Michler, Phys. Rev. B 93, 115308 (2016).

[46] J. H. Eberly and K. Wódkiewicz, J. Opt. Soc. Am. 67, 1252 (1977).

[47] A. Moelbjerg, P. Kaer, M. Lorke, and J. Mørk, Phys. Rev. Lett. 108, 017401 (2012).

[48] E. del Valle, A. Gonzalez-Tudela, F. P. Laussy, C. Tejedor, and M. J. Hartmann, Phys. Rev. Lett. 109, 183601 (2012).

[49] Y.-J. Wei, Y. He, Y.-M. He, C.-Y. Lu, J.-W. Pan, C. Schneider, M. Kamp, S. Höfling, D. P. S. McCutcheon, and A. Nazir, Phys. Rev. Lett. 113, 097401 (2014).

[50] S. M. Ulrich, S. Ates, S. Reitzenstein, A. Löffler, A. Forchel, and P. Michler, Phys. Rev. Lett. 106, 247402 (2011).

[51] A. J. Ramsay, A. V. Gopal, E. M. Gauger, A. Nazir, B. W. Lovett, A. M. Fox, and M. S. Skolnick, Phys. Rev. Lett. 104, 017402 (2010). 